\title{
Threshold recognition of phantom-contour objects requires constant contrast velocity
}

\author{
Patricia M. Kiely and Sheila G. Crewther \\ La Trobe University, Melbourne, Victoria, Australia \\ AND \\ DAVID P. CREWTHER \\ Swinburne University of Technology, Melbourne, Victoria, Australia
}

\begin{abstract}
Recognition of phantom objects - those with contours defined by rapid contrast reversal of adjacent fields of dark and light random dots - was investigated under conditions of abrupt or ramped onset and offset. Discrimination contrast thresholds were determined for a random-dot phantom letter in four possible orientations. For abrupt onset or offset, thresholds were almost independent of the duration of presentation time, over a range that varied tenfold, from 34-340 msec. However, when the onset and offset were shaped by a triangular envelope, thresholds were raised, so that form blindness occurred even when peak dot contrasts exceeded $60 \%$. Also under ramped onset and offset conditions, threshold contrast varied strictly linearly with stimulus duration in all subjects, suggesting a new construct - contrast velocity, the rate of change of contrast critical for phantomobject recognition.
\end{abstract}

Exquisite object discrimination as exhibited by the human visual system requires several early computational mechanisms, including the grouping of elements of the stimulus that are similar in orientation, motion, or other features; the segmentation of objects from the background; and preliminary matching of the partial object information against known target representations or against familiar token objects from iconic memory. Central to the process of grouping are the principles of "good continuation" and "common fate," a fact recognized by the Gestalt psychologists (see Lee \& Blake, 2001). These principles can work together in cluttered surroundings to improve the detection of objects.

The physiological mechanism by which contour extraction contributes to object recognition has been proposed to involve synchronization of the firing of cortical neurons (Eckhorn et al., 1988; reviewed by Gray, 1999). Although support for stimulus-dependent synchronous firing was initially derived from single-cell studies in the macaque monkey (Kreiter \& Singer, 1996), the evidence for synchrony affecting perception in human psychophysical studies has revolved around whether stimulus rather than neuronal synchrony can affect perceptual performance (a very different question). Thus, temporal phase differences can be used for texture discrimination (Fahle, 1993; Leonards, Singer, \& Fahle, 1996), and negative findings (Kiper, Gegenfurtner, \& Movshon, 1996) may possibly be due to the greater orientation bandwidth of the jittered stimulus grid used (Dakin \& Bex, 2002).
Following these early texture discrimination studies, Usher and Donnelly (1998) demonstrated that temporal asynchrony on a time scale less than visual integration time can strongly affect grouping; they claimed that binding is due to a global mechanism of grouping caused by synchronous neural activation. However, although the experimental findings have been replicated, their interpretation has been brought into question by experiments (Dakin \& Bex, 2002) demonstrating that contour binding associated with asynchronous presentation is disrupted by masking, image stabilization, and ramping of onset and offset. Although the effects of these interferences on the neural code are unknown, it is clear that the transients associated with asynchrony, either at onset or offset, are critical in contour detection and grouping. This finding has been refined (Beaudot, 2002), with stimulus asynchrony being shown to provide a benefit for detection only when the figure is presented before the background in the first cycle of stimuli.

Contour extraction by the visual system is all the more remarkable when the process is impoverished because of the self-masking provided by rapidly phase-reversing textures of light and dark dots, the boundaries between which are called phantom contours (Ramachandran \& RogersRamachandran, 1991). As such, the object-defining principle in this case is different from the one that operates for stimuli such as the commonly used snakes of nearly aligned Gabors not possessing sharp edges, although there is a common element of asynchrony automatically

D. P. Crewther, dcrewther@swin.edu.au 

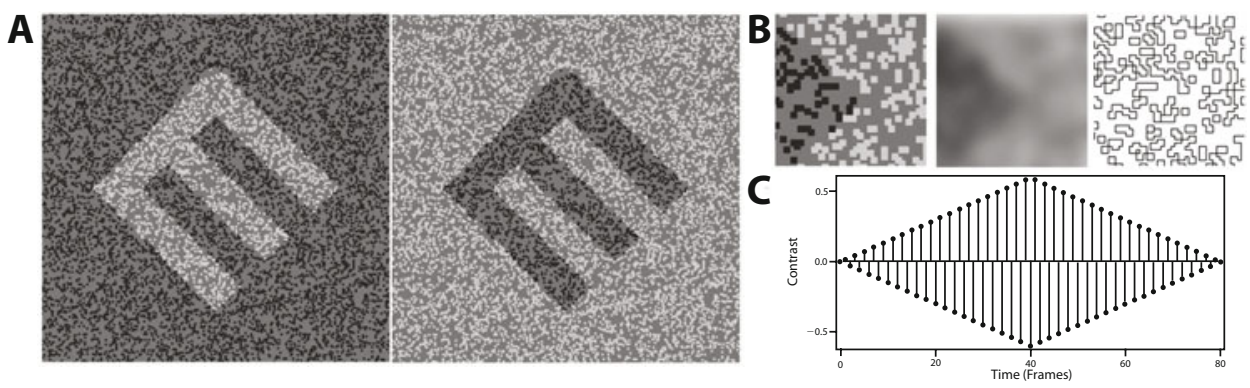

Figure 1. The stimulus consisted of a letter "E" (panel A) in which contrast reversed between the two forms shown, at a flicker rate of $58.5 \mathrm{~Hz}$ on a computer. The " $E$ " had a square profile and subtended $5^{\circ}$. For abrupt presentation, thresholds were measured for durations of $4,8,12,20,30$, and 40 frames (one frame $=$ $8.5 \mathrm{msec}$ ). For ramped presentation (both onset and offset), full-width-at-half-maximum durations were also $4,8,12,20,30$, and 40 frames. The letter was visible through texture generated by randomly distributed 1-mm dots on a mid-gray background (luminance $48 \mathrm{~cd} / \mathrm{m}^{2}$ ). Under such conditions, the edges (phantom contours) appear sharp and straight. (B) A close-up of the rightmost corner of the "E" shows the dark and light dots defining the letter. There is no continuous boundary or edge; an edge is visible, however, when low-pass filtered (16-pixel Gaussian blur), though high-frequency edge filters (Photoshop, Adobe Software) give no suggestion of the boundary. (C) Example of the temporal modulation of contrast (light-dot vs. dark-dot luminance) under a ramped condition (10 frames onset, 10 frames offset). A maximum Michelson contrast of approximately .6 is necessary to see the " $E$ " under such conditions.

present in the phase reversal of the phantom contours. It has been suggested that phantom contours activate a fast, sign-invariant, contour-extracting system, thought to be associated with the visual magnocellular system (Rogers-Ramachandran \& Ramachandran, 1998). Indeed, phantom-contour letter forms have been proposed as the basis of clinical tests of the magnocellular pathway (e.g., for glaucoma; see Simpson \& Flanagan, 1992). We have previously used such phantom letters to study the development of magnocellular processing in children, and have demonstrated an improvement in contrast sensitivity for phantom letters that plateaus at an age of 8-10 years (Barnard, Crewther, \& Crewther, 1998).

The likelihood that the process of object discrimination is aided by feedback from higher to lower cortical areas is suggested by physiological experiments that show that figure-background discrimination in areas V1 and V2 of the monkey is degraded when feedback connections are disrupted by cooling areas such as V5/MT (Bullier, 2001; Hupé et al., 1998). This suggests that such feedback connections are enhanced in importance as a consequence of the coarser scale of magnocellular system receptive fields and of the 10- to 20-msec prior entry of information to the cortex (Klistorner, Crewther, \& Crewther, 1997; Maunsell \& Gibson, 1992) that we term the magnocellular advantage (reviewed by Laycock, Crewther, \& Crewther, 2007).

We rationalized that the early transient response of magnocellular neurons could contribute timing information that informs the neural code for grouping and recognition. On the basis of previous grouping studies (Beaudot, 2002; Dakin \& Bex, 2002) indicating the importance of the first frame of presentation, we studied whether the contrast threshold for phantom-object discrimination depends strongly on the temporal profile of contrast onset and offset. Hence, we varied the duration of stimulus presentation under abrupt and ramped onset/offset conditions in order to establish whether object recognition can be impaired by removal of the initial transient response generated by magnocellular neurons.

\section{METHOD}

\section{Subjects}

Nine subjects (11-45 years of age) with normal or corrected-tonormal vision, all with experience of psychophysical testing, gave informed consent to participate in this experiment, as approved by the institutional ethics committee.

\section{Stimuli}

A capital letter "E" in one of four different orientations was defined by the illusory edge between a texture of lighter random dots (inside the "E") and a texture of darker dots (outside the "E"; see Figure 1A). When rapidly contrast-reversed (at $58.5 \mathrm{~Hz}$ ), the "E" is apparently defined by its edges - the so-called phantom contours (Ramachandran \& Rogers-Ramachandran, 1991). The "E" patterns were generated using Mathematica (Wolfram Research) with dots four pixels in size. The mean dot coverage was $31.8 \%$ of the area for both "E" and background.

The stimuli were delivered on an iMac computer (Apple Computer, Cupertino, CA) with luminances modulated around a mean value of $48 \mathrm{~cd} / \mathrm{m}^{2}$. The monitor frame refresh rate was $117 \mathrm{~Hz}$ $(8.4 \mathrm{msec} / \mathrm{frame})$. The stimulus presentation software was coded in Authorware Professional (Macromedia, Redwood City, CA), with time-critical routines programmed in $\mathrm{C}$

Two contrast envelopes were employed. The first comprised a square temporal envelope in which the contrast of the flickering pattern abruptly increased around the mid-gray background and stayed at a fixed level (with a maximum Michelson contrast of $90 \%$ between dark and light dots) for a set number of frames $(4,8,12$, 20,30 , or 40). This is referred to as the abrupt onset stimulus. The second stimulus employed a triangular ramped onset and offset envelope, in which the envelope increased in a linear fashion to a maximum value and then decreased to zero, again with a linear gradient (see Figure 1C). The letter and background were phase-reversing at $58.5 \mathrm{~Hz}$. This stimulus is referred to as the ramped stimulus. The onset and offset ramp durations were $4,8,12,20,30$, or 40 frames (i.e., lasting from 34 to $340 \mathrm{msec}$ ). 


\section{Procedure}

All subjects received training in the task prior to the gathering of experimental data. A forced choice procedure was used, such that subjects chose the orientation of the "E" from four options. Threshold (62.5\% correct) was estimated using an adaptive staircase (PEST) using a two-down one-up procedure. The staircase terminated at the tenth reversal, with the mean of the last four reversals used as the threshold contrast estimate. At least two threshold estimations were made for each stimulus duration setting. Normal room lighting was used, and subjects sat $60 \mathrm{~cm}$ from the computer monitor.

\section{RESULTS}

\section{Abrupt Onset}

Contrast thresholds for the individual subjects varied in a range of $1 \%-4 \%$ for exposure times between 4 and 40 frames (34-340 msec), with no significant change for the longer exposure times (see Figure 2A). That is, subjects were no better at recognizing the orientation of the letter with longer exposure, despite the increase by a factor of 10 in duration of presentation.

\section{Ramped Onset}

Threshold contrast demonstrated a high degree of linearity as a function of ramp duration for each subject. Amazingly, for the onset/offset with the longest duration, phantom letters with peak dot contrasts of over $60 \%$ failed to reach the discrimination threshold for most subjects (see Figure 2B). A linear regression of threshold as a function of duration showed individual correlation coefficients in excess of .9 in all cases (see Table 1).

This suggests definition of a quantity that we will term contrast velocity - the rate of change of threshold contrast with stimulus duration. When the individual data are replotted for contrast velocity as a function of duration, the constancy of contrast velocity across a wide range of durations is clearly evident (see Figure 2C, Table 1). An inspection of Table 1 indicates that the ordering of thresholds for abrupt-presentation stimuli among individuals is similar to that for contrast velocities. Indeed, rank correlation testing of the mean threshold contrast for the abruptpresentation trials against mean contrast velocity (for the ramped presentation trials) demonstrates a high correlation (Spearman's $\rho=.971$ ).

\section{DISCUSSION}

We draw three conclusions from these results. First, contrast sensitivity for abrupt presentation of phasealternating phantom figures does not improve with stimulus duration over the range from 34 to $340 \mathrm{msec}$, indicating that the initial one or two frames of presentation are critical for the process of recognition of phantom contours. Second, the increase of contrast threshold as a function of duration for the ramped stimulus presentation implies that images defined by phantom contours have to increase in contrast sufficiently rapidly if they are to be seen. Third, the precise linearity of slope (threshold vs. ramp duration; see Table 1) observed for all subjects suggests a fundamental property for the segmentation of images defined by phantom contours; we have called this property thresh-
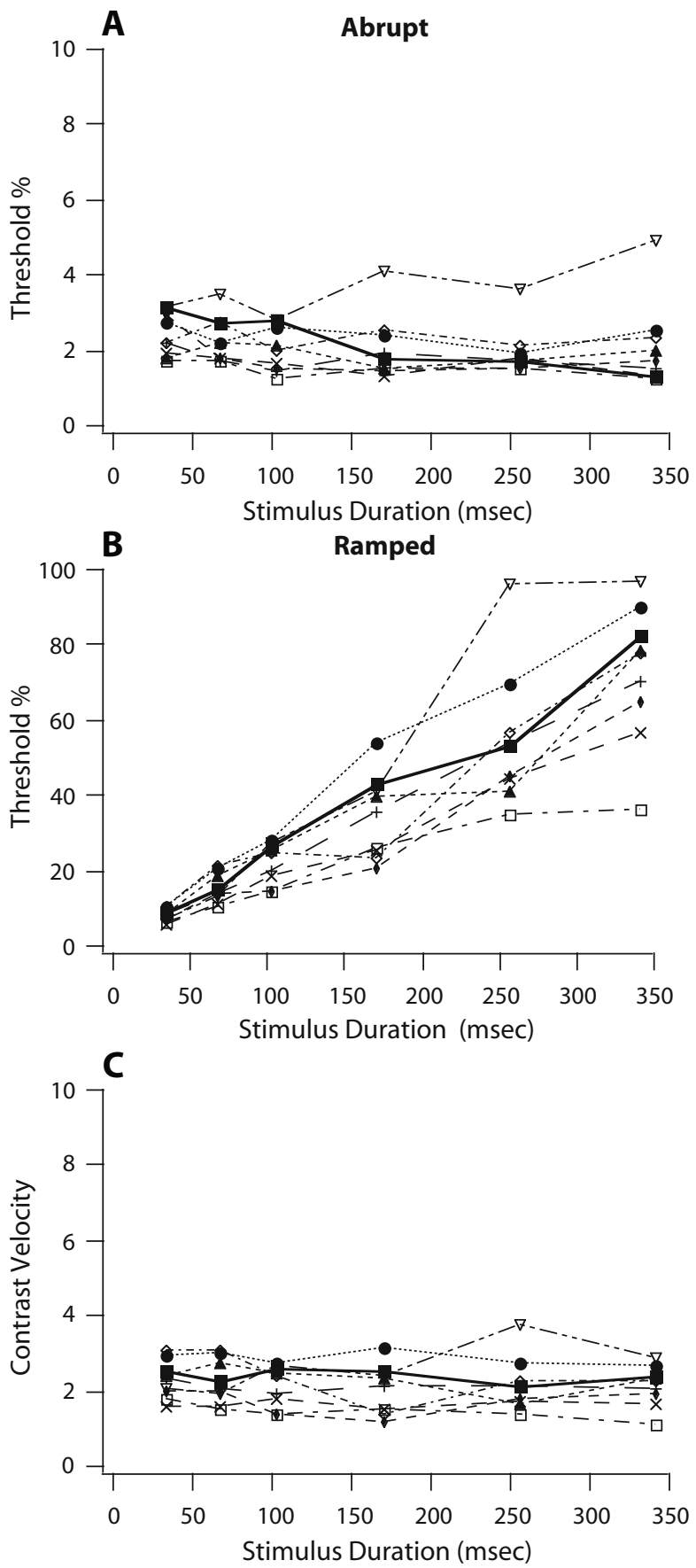

Figure 2. (A) Contrast threshold as a function of stimulus duration under abrupt-onset conditions for all subjects. A regression analysis showed that the mean slope is not significantly different from zero. (B) Threshold contrast (quoted as the Michelson contrast of light dots to dark dots) plotted as a function of ramp duration (full width at half maximum). All subjects showed linear functions of threshold versus duration and a high degree of consistency in slope. This suggests that the contrast velocity required for object recognition is a fundamental characteristic of human vision. Note that the ordinate scale is different from that in Figure 2A. (C) Contrast velocity (threshold contrast per unit stimulus duration in seconds), derived from Figure 2B and plotted against stimulus duration for each of the subjects. Contrast velocity was approximately constant for each subject over a range of stimulus duration that varied by a factor of 10 . 
Table 1

Mean Threshold Parameters for Abrupt and Ramped Onset

\begin{tabular}{cccc}
\hline & \multirow{2}{*}{$\begin{array}{c}\text { Abrupt Onset } \\
\text { Subject }\end{array}$} & \multicolumn{2}{c}{ Ramped Onset } \\
\cline { 3 - 4 } & Threshold \% & Slope $\left(\mathrm{sec}^{-1}\right)$ & Regression $r^{2}$ \\
\hline 1 & 2.2 & 2.39 & .982 \\
2 & 2.3 & 2.86 & .988 \\
3 & 1.8 & 2.29 & .922 \\
4 & 1.8 & 1.69 & .952 \\
5 & 2.2 & 2.39 & .921 \\
6 & 1.4 & 1.43 & .939 \\
7 & 2.9 & 3.13 & .97 \\
8 & 1.5 & 1.62 & .993 \\
9 & 1.7 & 2.08 & .998 \\
\hline
\end{tabular}

old contrast velocity. Such contrast velocity is a constant for each individual. Also, the high correlation observed between abrupt thresholds and contrast velocities across the group implies a relation in the process that leads to identification under the two conditions.

Our first finding is reminiscent of Beaudot (2002) and Dakin and Bex (2002), indicating that the first frame of a stimulus is important in a flickering foregroundbackground segmentation task and that the temporal asynchrony can be less than the integration time of the visual system (Usher \& Donnelly, 1998). However, the phantom contours differ from those used in other foreground-background temporal asynchrony experiments in that, for phantom contours, the foreground and background are presented simultaneously. The similarity resides in the fact that one can make the stimulus disappear by reducing the exposure period between phase reversals, just as the discrimination of foreground from background is eliminated as the asynchrony tends toward zero. That is, there needs to be sufficient time for some grouping to occur before the signal is canceled or masked. A second difference is that the phantom-contour stimulus is different from those employing snakes of nearly aligned Gabors, in that the phantom-contour percept is one that is purely edge-defined, whereas the snakes have smooth spatial transitions and are made visible by the relative alignment between the constituent Gabors.

Our finding that ramped onsets and offsets interfere with phantom-contour discrimination contrasts with that of Dakin and Bex (2002). They found that a ramped onset totally destroys target discrimination, and they interpreted this finding as evidence against the idea that temporal correlation among features can promote perceptual grouping (on the basis that ramping should not specifically disrupt a contour-binding scheme based on temporal synchrony). However, we found that orientation discrimination and letter recognition were possible with phantom "E"s under ramped onset and offset, but that the threshold was dependent on the rate of change of contrast in a fixed relationship - the contrast velocity. The fact that the stimuli were turned on and off over a varying number of frames, with threshold forming a fixed relation with duration, makes it unlikely that stimulus perception for phantom contours is strongly related to fixational eye movements (Wallis, 2005).
The effects we observed held across a wide range of ages among the subjects, and age was not a significant factor in determining threshold contrast velocity. It should be noted that the younger subjects had reasonable experience at performing psychophysical tasks, similar to that of psychology undergraduates. Indeed, our younger subjects showed variances that conformed with values obtained from adult subjects. In addition, the youngest subject was of an age at which the magnocellular pathway contribution to the VEP is reported to have reached mature values (Crewther, Crewther, Klistorner, \& Kiely, 1999).

Taken together, these results suggest that the initial temporal profile of stimulus presentation is critical for phantom-image discrimination. The lack of conscious perception at high nominal dot contrast when contrast is incremented slowly reinforces the notion that perception and discrimination require transient signaling.

\section{AUTHOR NOTE}

This work was supported by Grant A000937 from the Australian Research Council. The authors thank Jason Mattingley, Aina Puce, and Robert Hess for helpful criticisms of the manuscript. Correspondence relating to this article may be sent to D. P. Crewther, Brain Sciences Institute, Swinburne University of Technology, Hawthorn, Victoria 3122, Australia (e-mail: dcrewther@swin.edu.au).

\section{REFERENCES}

Barnard, N., Crewther, S. G., \& Crewther, D. P. (1998). Development of a magnocellular function in good and poor primary schoolage readers. Optometry \& Vision Science, 75, 62-68.

BEAUDOT, W. H. A. (2002). Role of onset asynchrony in contour integration. Vision Research, 42, 1-9.

Bullier, J. (2001). Integrated model of visual processing. Brain Research Reviews, 36, 96-107.

Crewther, S. G., Crewther, D. P., Klistorner, A., \& Kiely, P. M. (1999). Development of the magnocellular VEP in children: Implications for reading disability. Electroencephalography \& Clinical Neurophysiology, 49(Suppl.), 123-128.

DAKIN, S. C., \& BEX, P. J. (2002). Role of synchrony in contour binding: Some transient doubts sustained. Journal of the Optical Society of America A, 19, 678-686.

Eckhorn, R., Bauer, R., Jordan, W., Brosch, M., Kruse, W., MunK, M., \& Reitboeck, H. J. (1988). Coherent oscillations: A mechanism of feature linking in the visual cortex? Multiple electrode and correlation analyses in the cat. Biological Cybernetics, 60, 121-130.

FAHLE, M. (1993). Figure-ground discrimination from temporal information. Proceedings: Biological Sciences, 254, 199-203.

Gray, C. M. (1999). The temporal correlation hypothesis of visual feature integration: Still alive and well. Neuron, 24, 31-47, 111-125.

Hupé, J. M., James, A. C., Payne, B. R., Lomber, S. G., Girard, P., \& Bullier, J. (1998). Cortical feedback improves discrimination between figure and background by V1, V2 and V3 neurons. Nature, 394, 784-787.

Kiper, D. C., Gegenfurtner, K. R., \& Movshon, J. A. (1996). Cortical oscillatory responses do not affect visual segmentation. Vision Research, 36, 539-544.

Klistorner, A., Crewther, D. P., \& Crewther, S. G. (1997). Separate magnocellular and parvocellular contributions from temporal analysis of the multifocal VEP. Vision Research, 37, 2161-2169.

Kreiter, A. K., \& Singer, W. (1996). Stimulus-dependent synchronization of neuronal responses in the visual cortex of the awake macaque monkey. Journal of Neuroscience, 16, 2381-2396.

Laycock, R., Crewther, S. G., \& Crewther, D. P. (2007). A role for the "magnocellular advantage" in visual impairments in neurodevelopmental and psychiatric disorders. Neuroscience \& Biobehavioral Reviews, 31, 363-376.

LEE, S. H., \& BLAKE, R. (2001). Neural synergy in visual grouping: 
When good continuation meets common fate. Vision Research, 41, 2057-2064.

LeOnards, U., Singer, W., \& FAhLE, M. (1996). The influence of temporal phase differences on texture segmentation. Vision Research, 36, 2689-2697.

Maunsell, J. H. R., \& Gibson, J. R. (1992). Visual response latencies in striate cortex of the macaque monkey. Journal of Neurophysiology, 68, 1332-1344

RAMACHANDRAN, V. S., \& Rogers-Ramachandran, D. C. (1991). Phantom contours: A new class of visual patterns that selectively activates the magnocellular pathway in man. Bulletin of the Psychonomic Society, 29, 391-394.

Rogers-Ramachandran, D. C., \& Ramachandran, V. S. (1998). Psy- chophysical evidence for boundary and surface systems in human vision. Vision Research, 38, 71-77.

Simpson, T., \& Flanagan, J. G. (1992). A contour illusion letter test [Abstract]. Investigative Ophthalmology \& Visual Science, 33(4), 1385.

Usher, M., \& Donnelly, N. (1998). Visual synchrony affects binding and segmentation in perception. Nature, 394, 179-182.

WALLIS, G. (2005). A spatial explanation for synchrony biases in perceptual grouping: Consequences for the temporal-binding hypothesis. Perception \& Psychophysics, 67, 345-353.

(Manuscript received June 20, 2005; revision accepted for publication January 13, 2007.) 